\title{
What do we really know about the magnetic fields of the Milky Way?
}

\author{
Richard Wielebinski \\ Max-Planck-Institut für Radioastronomie, Auf dem Hügel 69, 53121 Bonn, Germany \\ email: rwielebinski@mpifr-bonn.mpg.de
}

\begin{abstract}
We have several methods of measuring magnetic fields in the Milky Way. We can study optical polarization, radio polarization, rotation measures of pulsars and extragalactic radio sources as well as include Zeeman results. Each of the above mentioned methods was at times used to make a model of the magnetic fields of the Milky Way. However one or two of the data sets by themselves cannot tell us the whole story. Any model of the magnetic fields must be able to fit all the observational results. At the present time a lot of progress has been made. We have increased our data bases in most of the observational areas. However a robust model of the magnetic field of the Milky Way has not yet emerged. We must possibly wait to the era of SKA.
\end{abstract}

Keywords. Galaxies: Milky Way - galaxies: magnetic fields - galaxies: structure - ISM: magnetic field

\section{Introduction}

We have been studying the magnetic fields of the Milky Way ever since the first optical polarization observations were published. Radio observations confirmed the optical results and gave a new impetus to the study of cosmic magnetic fields. Extragalactic sources became potential probes of the magnetic fields in the Milky Way. Finally pulsars became the probes par excellence for the determination of Milky Way's magnetic field. The Zeeman effect, the logical method of observing magnetic fields, has succeeded only in molecular clouds so far.

Models of the magnetic fields in the Milky Way followed the observational results. From the very beginning it was clear that the magnetic field was directed along the spiral arms. Optical polarization data suggested that this pertained only to the nearby magnetic fields, i.e. $l<1 \mathrm{kpc}$. The 'B' vectors were generally aligned along the Galactic plane with some suggestion of extensions along the North Polar Spur. The general discussion followed the original description of the spiral structure of the Galaxy proposed by Georgelin \& Georgelin (1976). The discussion then evolved around the question whether the magnetic fields of the Milky Way were bi-symmetrical or axi-symmetrical. The bi-symmetrical idea bases on the early suggestion by Piddington (1964) that compression of a primordial field by a rotating galaxy would create such a magnetic field pattern. The alternative interpretation was offered by the dynamo theory (e.g. Parker 1955; Steenbeck \& Krause 1969) especially after the multimode extension of the dynamo process was published (Baryshnikova, Shukurov, Ruzmaikin, et al. 1987). In this theory a multiplicity of modes is possible, including the axi-symmetric mode. In the present contribution I will discuss the recent observational results and conclude that we still have no robust model of the magnetic fields of the Milky Way. I will also point out that any model must satisfy the data available from the different observational methods. 


\section{Optical polarization}

After the early observations, dating back to 1949, various observers collected data on the polarization of stars. A synthesis of all the available data was made by Mathewson $\&$ Ford (1970) that showed the general structure of the nearby magnetic field. The local magnetic field was aligned along the Galactic Plane with some additional loops that indicated local objects (large Supernova Remnants). More recent catalogues collected by Heiles (2000) increased the number of objects but did not go much beyond the original limitation of observing the nearby magnetic field.

\section{Radio continuum polarization}

The polarization of the diffuse radio emission that was finally discovered in 1962 had a long period of quiescence after the surveys of Wielebinski \& Shakeshaft (1964), Mathewson \& Milne (1965) and Brouw \& Spoelstra (1976) have been published. The follow-up investigation by Spoelstra (1984) concluded that the Rotation Measure (RM) in the ISM towards $l=140^{\circ}$ was low, $R M<18 \mathrm{rad} \mathrm{m}^{-2}$. The realization that polarization changes dramatically with higher angular resolution due to high RM (e.g. Wieringa, de Bruyn, Jansen, et al. 1993; Uyanıker, Fürst, Reich, et al. 1999) led to new work that has added a lot of significant observations. Radio polarization observations at higher frequencies allowed investigations deeper in the Galaxy, showing that even at the Galactic centre continuum polarization can be observed (e.g. Seiradakis, Lasenby, Yusef-Zadeh, et al. 1985). The northern sky was mapped in polarization by Wolleben, Landecker, Reich, et al. (2006) and the southern sky by Testori, Reich \& Reich (2008) both with good angular resolution. These surveys showed that the Faraday effects are large and not only confined to the narrow Galactic plane (see the contribution of W. Reich \& P. Reich, these Proceedings). This was also confirmed by Gaensler, Madsen, Chatterjee, et al. (2008) who analysed pulsar dispersion measure and the diffuse $\mathrm{H} \alpha$ emission. The three-year WMAP data (Page, Hinshaw, Komatsu, et al. 2007) gave us new insight of the radio polarization distribution at high frequencies. The new thermal electron model of Cordes \& Lazio (2002) was also available. As a result of these surveys a simulation was made by Sun, Reich, Waelkens, et al. (2008) which is an advance on previous such investigations. The work of Sun, Reich, Waelkens, et al. (2008) suggests an axi-symmetric magnetic field of the Galaxy. To confirm this result we need a dense grid of RMs (an RM all-sky survey) with all the anomalous values due to individual HII regions sorted out.

\section{Extragalactic radio sources}

The collection of data on the Rotation Measure of extra-galactic radio sources (EGRS) has a long tradition. The survey of Simard-Normandin \& Kronberg (1980) was used to model the magnetic fields in the Milky Way. In this work the first identification of a magnetic field reversal was made. The discussion about reversals has been taken up by a number of authors, e.g. Vallée $(1983,2005)$. However in the data base of SimardNormandin \& Kronberg (1980) very few EGRS were observed through the plane of the Galaxy, where the magnetic fields are concentrated. This investigation of RM of the EGRS in the Galactic plane was taken up by Brown \& Taylor (2001); Brown et al. (2003, 2007). The northern sky data shows a rather regular distribution of RMs towards the Perseus arm with some rather high values of $\mathrm{RM}$, e.g. $\mathrm{RM} \sim 700 \mathrm{rad} \mathrm{m}^{-2}$. The southern sky survey of EGRS in the Galactic plane showed that values of up to RM $\sim 1000 \mathrm{rad} \mathrm{m}^{-2}$ are observed. Also the observed reversals of the sign of the RM led Brown, Haverkorn, Gaensler, et al. (2007) to conclude that there is possibly a magnetic field reversal in the inner Galaxy. The study of the RM of EGRS towards Galactic centre by Roy, Pramesh 
Rao \& Subrahmanyan (2008) led to the suggestion of a bi-symmetrical magnetic field. To confirm this interpretation we need a much denser sampling of sources.

\section{Pulsars}

Pulsars give an excellent method of studying magnetic fields since the combination of the Dispersion Measure DM and the RM gives us a value of the mean magnetic field along the line of sight. From the DM and a model of electrons (e.g. Cordes \& Lazio 2002) a distance of the pulsar can be determined. The discussion about the magnetic fields of the Galaxy based on pulsar observations dates back to a number of authors (e.g. Rand \& Kulkarni 1989; Han \& Qiao 1994). The addition of new pulsar observations let to even more detailed models (e.g. Han, Manchester, Lyne, et al. 2002, 2006) suggesting a number of large-scale reversals as could be expected in a bi-symmetrical system. Recently however a number of new investigations questioned this model. The field reversal beyond the Perseus arm was shown (Mitra, Wielebinski, Kramer, et al. 2003) to be due to two pulsars observed behind an HII region, where the magnetic field is reversed. Also studies of further pulsars by Noutsos, Johnston, Kramer, et al. (2008) questioned the accepted interpretation. Men, Ferrière \& Han (2008) investigated the available pulsar data and ruled out all the simple models that have been published to date.

\section{The Zeeman effect}

The Zeeman effect is the method par excellence to measure magnetic fields. However we are limited here to dense molecular clouds in the Milky Way (e.g. Heiles \& Crutcher 2005). A recent investigation of the Zeeman results by Han \& Zhang (2007) suggests that molecular clouds "remember" the direction of the local magnetic field. Since we do not know exactly the real directions of the local magnetic field we must wait until more data becomes available.

\section{Discussion with some conclusions}

There are many local reversals seen in the pulsar and EGRS data sets. Some of the reversals seem to be associated with HII regions. A large, but possibly local reversal is seen towards $l=75^{\circ}$. Most of the pulsars that show this reversal are at a distance of $d \sim 1 \mathrm{kpc}$. This would be a local rather than large scale reversal. There are directions where the agreement between pulsar and EGRS data is good. On the other hand serious disagreement is seen, for instance in the direction $l=10^{\circ}, b=25^{\circ}$. The values of RMs in the anti-centre region are not very different to the RMs seen towards the Galactic centre. Also the pulsars and EGRS in the Galactic plane seem to be seen through similar Faraday screens - are these effects all local? This would also put the standard method of estimation of pulsar distances in question. It would also suggest that Faraday Rotation takes place in localised regions of high magnetic fields and that we cannot expect to map the magnetic fields of the Milky Way by these methods.

I have collected all the recently published literature pertaining to modelling of the magnetic fields of the Milky Way and have pointed out the limitations of the conclusions reached by many authors. An important fact that must be remembered is that all the different data sets must agree with a proposed robust model of the magnetic field of the Milky Way. It is not realistic to build up a model using only pulsars but disregarding radio continuum polarization data. We also must have a good sampling of the sources across the sky before we can make robust models. In particular we have insufficient data on the RM of southern EGRS. Pulsars are concentrated close to the Galactic plane and the inner Galaxy. The observations of RM of EGRS along the Galactic plane is still incomplete. There is a great need of all-sky surveys (with polarization) in the $\lambda \sim 6 \mathrm{~cm}$ 
wavelength range. We must have information (all-sky surveys) of the RM of the Galactic radio continuum with good angular resolution. Some of these 'needs' will be addressed by new projects like LOFAR or SKA. Some projects must be executed by dedicated special instruments. One of the 'small' problems that must be addressed is the absolute calibration of polarization surveys.

\section{References}

Baryshnikova, I., Shukurov, A., \& Ruzmaikin, A., et al. 1987, A\& A 177, 27

Brouw, W. N. \& Spoelstra, T. A. Th. 1976, A\& AS 26, 129

Brown, J. C. \& Taylor, A. R. 2001, ApJ 563, L31

Brown, J. C., Taylor, A. R., Wielebinski, R., \& Müller, P. 2003, ApJ 592, L29

Brown, J. C., Haverkorn, M., \& Gaensler, B. M., et al. 2007, ApJ 663, 258

Cordes, J. M. \& Lazio, T. J. W. 2002, astro-ph/0207156v2

Gaensler, B. M., Madsen, G. J., \& Chatterjee, S., et al. 2008, astro-ph:0808.2550

Georgelin, Y. M. \& Georgelin, Y. P. 1976, A\&A 49, 57

Han, J. L. \& Qiao, G.J. 1994, $A \& A$ 288, 759

Han, J. L. \& Zhang, J. S. 2007, A\&A 464, 609

Han, J. L., Manchester, R. N., Lyne, A, G., \& Qiao, G. J. 2002, ApJ 570, L17

Han, J. L., Manchester, R. N., \& Lyne, A. G., et al. 2006, ApJ 642, 868

Heiles, C. 2000, AJ 119, 923

Heiles, C. \& Crutcher, R. 2005, in: R. Wielebinski \& R. Beck (eds.), Cosmic Magnetic Fields, LNP 664 (Heidelberg: Springer), p. 137

Mathewson, D. S. \& Ford, V. L. 1970, MemRAS 74, 139

Mathewson, D. S. \& Milne, D. K. 1965, Australian J. Phys. 18, 635

Men, H., Ferrière \& Han, J. L. 2008, A $\mathscr{E} A$ 486, 819

Mitra, D., Wielebinski, R., Kramer, M., \& Jessner, A. 2003, A\&̋A 398, 993

Noutsos, A., Johnston, S., \& Kramer, M., et al. 2008, MNRAS 386, 1881

Page, L., Hinshaw, G., \& Komatsu, E., et al. 2007, ApJS 170, 335

Parker, E.N. 1955, ApJ 122, 293

Piddington, J. H. 1964, MNRAS 128, 345

Rand, R. J. \& Kulkarni, S. R. 1989, ApJ 343, 760

Roy, S., Pramesh Rao, A. \& Subrahmanyan, R. 2008, A\&A 4 478, 435

Seiradakis, J. H., Lasenby, A. N., \& Yusef-Zadeh, F., et al. 1985, Nature 317, 697

Simard-Normandin, M. \& Kronberg, P. P. 1980, ApJ 242, 74

Spoelstra, T. A. Th. 1984, A\& A 135, 238

Steenbeck, M. \& Krause, F. 1969, AN 291, 49

Sun, X. H., Reich, W., Waelkens, A., \& Enßlin, T. A. 2008, A\& A 477, 573

Testori, J. C., Reich, P., \& Reich, W. 2008, A\&A 484, 733

Uyanıker, B., Fürst, E., \& Reich, W., et al. 1999, A\&AS 138, 31

Vallée, J. P. 1983, A\&A 124, 147

Vallée, J. P. 1996, A\&A 308, 433

Vallée, J. P. 2005, ApJ 619, 297

Wielebinski, R. \& Shakeshaft, J. R. 1964, MNRAS 128, 19

Wieringa, M. H., de Bruyn, A, G., \& Jansen, D., et al. 1993, A\&SA 268, 215

Wolleben, M., Landecker, T. L., Reich, W., \& Wielebinski, R. 2006, A\&SA 448, 411

\section{Discussion}

STIL: Comment: The high rotation-measure (RM) pulsar near the region $\ell=5 b=+24$ coincides with an HII region around the runaway O star $\zeta$ Oph. The HII region causes bandwidth depolarization of NVSS sources, and therefore has a high rotation measure.

Wielebinski: Yes, you must look in detail in such areas. The RM varies rapidly even on scales of 1 arcmin - as seen in the CGPS - aee poster P5.10. 